\title{
Systemic and Local Phenotypes of Barium Chloride Induced Skeletal Muscle Injury in Mice
}

\author{
Hee-Won Jung ${ }^{1}$, Jin-Hyuk Choi ${ }^{2}$, Taehee Jo ${ }^{1}$, Hyemi Shin ${ }^{2}$, Jae Myoung Suh ${ }^{1,2}$ \\ ${ }^{1}$ Graduate School of Medical Science and Engineering, Korea Advanced Institute for Science and Technology, Daejeon, Korea \\ ${ }^{2}$ Biomedical Science and Engineering Interdisciplinary Program, Korea Advanced Institute for Science and Technology, Daejeon, Korea
}

Corresponding Author:

Jae Myoung Suh, $\mathrm{PhD}$

https://orcid.org/0000-0001-8097-4662

Graduate School of Medical Science

and Engineering, Korea Advanced Institute for Science and Technology, 291 Daehak-ro, Yuseong-gu, Daejeon 34141, Korea

E-mail:.jmsuh@kaist.ac.kr

Received: March 20, 2019

Revised: May 12, 2019

Accepted: May 25, 2019

\begin{abstract}
Skeletal muscle regeneration in mice has traditionally been studied using local freeze burn or snake venom injection models. More recently, a barium chloride $\left(\mathrm{BaCl}_{2}\right)$-induced muscle injury model has been established and is gaining popularity due to the relatively simple procedure and accessibility to required reagents. Here we sought to characterize the local and systemic effects of $\mathrm{BaCl}_{2}$-induced muscle injury. For this study, a $1.2 \%$ $\mathrm{BaCl}_{2}$ solution was locally administered to the tibialis anterior (TA) muscle and local and systemic phenotypes were analyzed at different timepoints. When $50 \mu \mathrm{L}$ of the solution was injected unilaterally in the TA muscle, no mortality was observed. However, when 100 $\mu \mathrm{L}$ of the solution was injected, $50 \%$ of the mice died within $24 \mathrm{~h}$. Serum analysis of the mice injected with $50 \mu \mathrm{L}$ of $\mathrm{BaCl}_{2}$ solution at days 1 and 7 revealed changes resembling rhabdomyolysis. At day 1 post-injection of $50 \mu \mathrm{L}$ of the $\mathrm{BaCl}_{2}$ solution, acute suppurative inflammation was observed in gross examination of the TA muscle, while extensive hemorrhagic necrosis was revealed on histological examination. At day 7 , regenerated myofibers with centralized nuclei appeared with the resolution of acute inflammatory infiltration and the muscle tissue displayed molecular signatures consistent with myofiber differentiation. The overall muscle injury and regeneration phenotypes in the $\mathrm{BaCl}_{2}$-induced muscle injury model were similar to those of the well-established freeze burn or snake venom injection models. Taken together, the $\mathrm{BaCl}_{2}$-induced muscle injury model is comparable to conventional muscle injury and regeneration models, with considerations for possible systemic effects. (Ann Geriatr Med Res 2019;23:83-89)
\end{abstract}

Key Words: Muscle regeneration, Barium chloride, Phenotype

\section{INTRODUCTION}

In living organisms, tissue injury and repair processes have evolved to restore tissue homeostasis as a response to damage and insults arising from both extrinsic and intrinsic factors. ${ }^{1)}$ Unlike many non-vertebrates and some vertebrates such as salamanders, the tissue repair capabilities in mammals are relatively limited. Tissue regeneration processes in vertebrates require adult stem cells, which are maintained and regulated through complex interactions between the stem cells and cellular and humoral components of the surrounding milieu, ${ }^{2)}$ and these interactions are often affected by biological events such as aging. In clinical medicine, understanding how appropriate tissue regeneration processes occur without organ function impairment and systemic adverse effects has important implications in the prognosis and treatment of disease. ${ }^{3)}$

Skeletal muscle is one of the most widely studied tissues in regenerative biology due to its accessibility to experimental injury models and its ability to regenerate efficiently in a relatively short timeframe of 14 days in laboratory mice. ${ }^{4,5)}$ Furthermore, the clinical importance of alterations in skeletal muscle in older adults provides strong motivation to study skeletal muscle regeneration processes within the context of aging. ${ }^{6}$ In the pathophysiology of sarcopenia, a common geriatric condition defined by reduced muscle strength, performance and/or muscle mass, multifaceted causes including sedentary lifestyle, poor nutrition, hormonal changes with aging, and systemic inflammation have been reported to accelerate muscle wasting in older adults. ${ }^{7-10)}$ Biologically, one of the contributing factors for sarcopenia is a decreased ability of aged muscle to regenerate following injury, ${ }^{11,12)}$ prompting research to study the effects of aging on muscle stem cells and its paracrine milieu in muscle regeneration processes after injury.

In studying the skeletal muscle regeneration process, animal models commonly use direct injuries to individual 
muscles such as the tibialis anterior (TA) or extensor digitorum longus (EDL). ${ }^{13,14)}$ Historically, the local injection of snake venoms such as cardiotoxin or notexin, which induce prolonged depolarization and disruption of cellular membranes, has been a popular and common method to induce muscle injury and regeneration. ${ }^{5)}$ In the typical course of this muscle injury model, exudative acute inflammation with mononuclear cell infiltration occurs 12-24 h after toxin injection, with subsequent myogenic proliferation and new myotube formation with centralized nuclei occurring 5-6 days after the initial injury. Thereafter, myofiber maturation occurs until 10-14 days after the initial injury. ${ }^{5,15)}$

A skeletal muscle injury model provided by the local injection of barium chloride solution $\left(\mathrm{BaCl}_{2}\right)$ has recently been established and is gaining popularity as a model for studying muscle regeneration. ${ }^{16)} \mathrm{BaCl}_{2}$ has advantages over previous reagents to induce muscle injury due to its ease of purchasing and transporting without the regulatory restrictions that apply to snake venoms. $\mathrm{BaCl}_{2}$ has been traditionally used as a potassium channel blocker in experimental physiology ${ }^{17)}$ and is thought to induce myonecrosis in muscle tissue by eliciting prolonged depolarization of myofibers, analogous to snake venoms. However, compared to snake venoms, the detailed local effects of $\mathrm{BaCl}_{2}$ across a time course, including both gross and microscopic morphology, have been less studied. In addition, although its systemic toxicity ${ }^{18)}$ (median lethal dose [LD50] of 19.2 $\mathrm{mg} \mathrm{Ba} / \mathrm{kg}$ in ICR mice) associated with arrhythmogenic effects has been reported, ${ }^{19)} \mathrm{BaCl}_{2}$-induced muscle injury models have been largely considered a locally specific method for the study of muscle regeneration. Therefore, this study sought to assess the acute systemic effects after $\mathrm{BaCl}_{2}$ injection in mouse TA muscle along and to perform a detailed analysis of local phenotypes during injury repair along a temporal scale.

\section{MATERIALS AND METHODS}

\section{Animals}

The animal experiments in this study were approved by the Institutional Animal Care and Use Committee at the Korea Advanced Institute of Science and Technology (KAIST), Daejeon, Republic of Korea (approval number of KA2015-34). Male C57BL6/J mice were maintained in a specific pathogen-free animal facility at KAIST and were housed at room temperature $\left(24^{\circ} \mathrm{C}\right)$ under a $12 \mathrm{~h}$ light/dark cycle. All mice were allowed access to water and a standard chow diet ad libitum. Mice between 12 and 15 weeks of age were used for the experiments.

\section{Muscle Injury Procedure}

All muscle injury procedures were performed under inhaled anesthesia using sevoflurane with a mixture of oxygen with room air. In studying acute survival associated with $\mathrm{BaCl}_{2}$ injection, $100 \mu \mathrm{L}$ or $50 \mu \mathrm{L}$ of a $1.2 \% \mathrm{BaCl}_{2}$ solution dissolved in phosphate buffered saline (PBS) was injected to the TA muscles of sedated mice. Since we anecdotally observed that mice inadvertently given a higher dose of $\mathrm{BaCl}_{2}$ solution deceased one day after injection, we selected $100 \mu \mathrm{L}$ and $50 \mu \mathrm{L}$ doses for survival analysis. For the study of the local phenotypes and levels of serum markers, $50 \mu \mathrm{L}$ of a $1.2 \% \mathrm{BaCl}_{2}$ solution dissolved in PBS was injected into the TA muscles of sedated mice. The contralateral hindlimbs served as untreated controls.

\section{Blood Chemistry}

At day 0 , as a control group before $\mathrm{BaCl}_{2}$ injection, and at days 1 and 7 after $\mathrm{BaCl}_{2}$ injection, whole blood was collected in serum-separating tubes by cardiac puncture. From the separated serum after centrifugation, levels of chemical markers including aspartate aminotransferase (AST), alanine aminotransferase (ALT), total cholesterol, creatinine kinase (CK), creatinine, glucose, C-reactive protein (CRP), and lactate dehydrogenase (LDH) were measured using a Cobas 8000 modular analyzer system (Roche, Basel, Switzerland) according to the manufacturer's protocol.

\section{Tissue Harvest, Histological Analysis, and RNA Extraction}

Upon euthanasia and sacrifice of the mice, the TA muscles were quickly harvested at 1, 3, 5, 7 days after $\mathrm{BaCl}_{2}$ injection. For histological analysis, the muscles were fixed in neutral buffered formalin overnight and then processed and embedded in paraffin for hematoxylin and eosin staining. Total RNA from TA muscles 7 days after $\mathrm{BaCl}_{2}$ injection was extracted using TRIzol (Invitrogen, Carlsbad, CA, USA) according to the manufacturer's protocol. After extraction, RNA quality was assessed on a Bioanalyzer (Agilent Technologies, Palo Alto, CA, USA). RNA samples with RNA integrity numbers (RINs) above 7.0 were used for RNA sequencing.

\section{RNA Sequencing}

For RNA sequencing, libraries were prepared with an Illumina TruSeq Sample Preparation Kit and sequenced on a NovaSeq6000 system (Illumina, San Diego, CA, USA) with multiplexing to produce more than 40 million reads per sample. The reads were converted to fragments per kilobase of transcript per million (FPKM) after alignment to the reference mouse genome. We considered differentially expressed genes with at least two-fold changes in expression values between groups.

\section{Protein Extraction and Western Blot Analysis}

Protein lysates were prepared from TA muscles in control mice and test mice 1, 4, and 7 days after $\mathrm{BaCl}_{2}$ injection using RIPA lysis buffer (Thermo Fischer Scientific, Wilmington, DE, USA) containing a protease inhibitor cocktail. Protein concentrations were measured using a BCA Protein Assay Kit (Thermo Fisher Scientific); the samples were denatured in sodium dodecyl sulfate (SDS) loading 
buffer, separated by $12 \%$ SDS-polyacrylamide gel electrophoresis (SDS-PAGE) and transferred to polyvinylidene difluoride (PVDF) membranes. The transferred membranes were examined through Ponceau S staining. Following blocking with 3\% bovine serum albumin for $30 \mathrm{~min}$, the membranes were incubated overnight at $4^{\circ} \mathrm{C}$ with anti$\beta$-catenin (1:1,000, \#8480), anti-phospho-ERK $(1: 1,000$, \#9101), anti-ERK (1:1,000, \#9102), anti-phospho-S6 (1:1,000, \#2211), anti-S6 (1:1,000, \#2217), and anti- $\alpha-$ tubulin (1:2,000, \#2144) antibodies. After incubation with horseradish peroxidate (HRP)-conjugated host-specific secondary antibody, the resulting blot was visualized on a ChemiDoc XRS+ system (Biorad, Hercules, CA, USA). All antibodies were purchased from Cell Signaling Technology (Danvers, MA, USA).

\section{Statistical Analysis}

For statistical comparison, values between groups were compared using independent t-tests. For acute systemic toxicity, survival analysis was performed with log-rank tests and Kaplan-Meier plots. Statistical analysis was performed using GraphPad Prism version 6 (GraphPad Software, San Diego, CA, USA), with two-sided p-values $<0.05$ considered statistically significant.

\section{RESULTS}

\section{Systemic Effects of the $\mathrm{BaCl}_{2}$-Induced Muscle Injury Model}

We first assessed for possible acute systemic toxicity with local $\mathrm{BaCl}_{2}$ injection of TA muscle. Upon injection of 50 $\mu \mathrm{L}$ or $100 \mu \mathrm{L}$ of a $1.2 \% \mathrm{BaCl}_{2}$ solution, four of eight mice in the $100 \mu \mathrm{L}$ group died within $24 \mathrm{~h}$ after injection, while none of the eight mice in the $50 \mu \mathrm{L}$ group died (Fig. 1A). Observation of the mice until 15 days after injection revealed no subacute mortality after the initial $24 \mathrm{~h}$ in both groups.

Gross examination of separated serum color showed a bright red color at days 1 and 7 after injection of $50 \mu \mathrm{L}$ $\mathrm{BaCl}_{2}$ to the TA muscle (Fig. 1B), indicative of myoglobinemia. Accordingly, significant increases in serum CK and LDH levels were observed at days 1 and 7, compared to those in the uninjected controls (Fig. 1C). There were similar patterns of increased LDH and AST at days 1 and 7, tracking with serum CK levels, suggesting that the increase in AST and LDH was due to acute myonecrosis. There was no statistical difference in ALT creatinine levels between the control, day 1, and day 7 groups (Fig. 1C). The serum CRP levels were less than $0.5 \mathrm{mg} / \mathrm{L}$ in all three groups (data not shown).


B
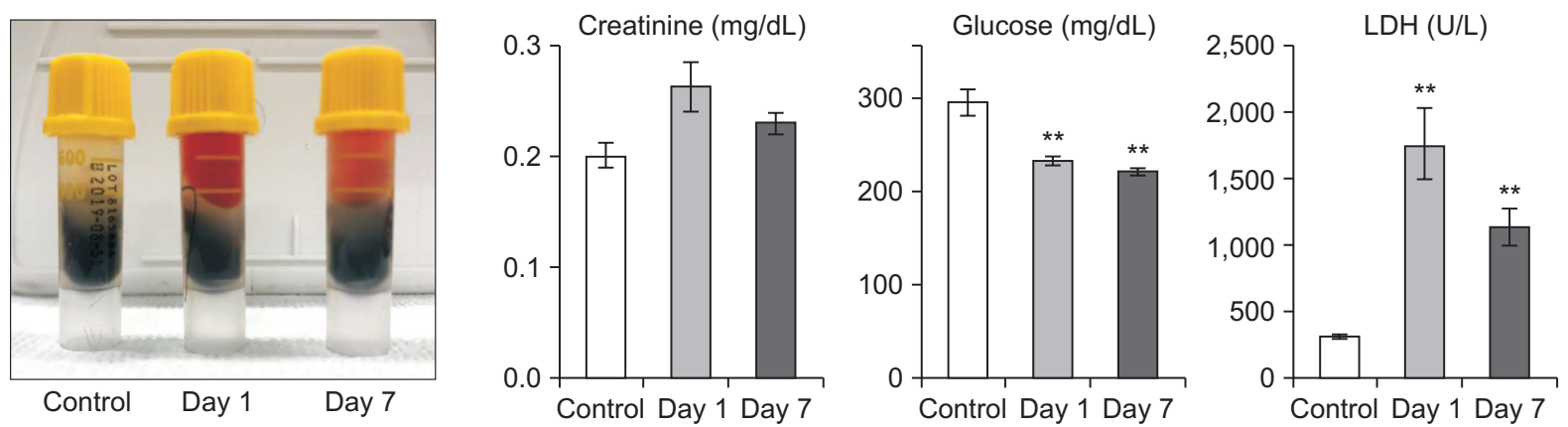

Fig. 1. Systemic phenotypes of the barium chloride $\left(\mathrm{BaCl}_{2}\right)$-induced muscle injury model. (A) Kaplan-Meier survival plot of mice that underwent $\mathrm{BaCl}_{2}$ injection of the ipsilateral tibialis anterior muscle ( $\mathrm{n}=8$ for each group, log-rank $\mathrm{p}$-value $<0.001$ ). (B) Red-colored serum at days 1 and 7 after $\mathrm{BaCl}_{2}$ injection after centrifugation. (C) Analysis of serum biochemical markers from mice at days 1 and 7 after $\mathrm{BaCl}_{2}$ injection, with uninjured mice (control). ALT, alanine aminotransferase; AST, aspartate aminotransferase; CK, creatinine kinase; $\mathrm{LDH}$, lactate dehydrogenase. ${ }^{* *} \mathrm{p}<0.01,{ }^{* * * *} \mathrm{p}<0.001$. 


\section{Local Gross and Microscopic Morphology in the $\mathrm{BaCl}_{2}-$ Induced Muscle Injury Model}

To examine the temporal changes in the gross and histological morphologies of TA muscle after $\mathrm{BaCl}_{2}$-induced muscle injury, we harvested TA muscle at 1, 3, 5, and 7 days after local $\mathrm{BaCl}_{2}$ injection $(50 \mu \mathrm{L})$ and performed hematoxylin and eosin staining (Fig. 2). Grossly, hemorrhagic edema peaked at day 1 and gradually subsided during the regeneration process. Histologically, hemorrhagic inflammation with pronounced exudation of red blood cells was observed at day 1 and mononuclear infiltration peaked at day 3. At day 5, large numbers of small myofibers with centralized nuclei appeared and mononuclear infiltration was mostly cleared at day 7 .

\section{Molecular Signatures of Muscle Regeneration in the $\mathrm{Bacl}_{2}$-Induced Muscle Injury Model}

We assessed the gene expression profile of muscle regeneration from RNA sequencing data comparing regenerating TA muscles at 7 days after $\mathrm{BaCl}_{2}$-induced injury to uninjured muscles ( $n=4$ for each group). The expression profiles of genes reflecting proliferating cells showed mixed results, with Pax7 and Myf5 significantly increased at day 7 but Myod1, Foxk1, Des showing no significant difference when compared to uninjured muscles (Table 1). In contrast, genes reflecting differentiating muscle stem cells (satellite cells) including Fgfr4, Myh3, Atp2a2, Tnnt1,

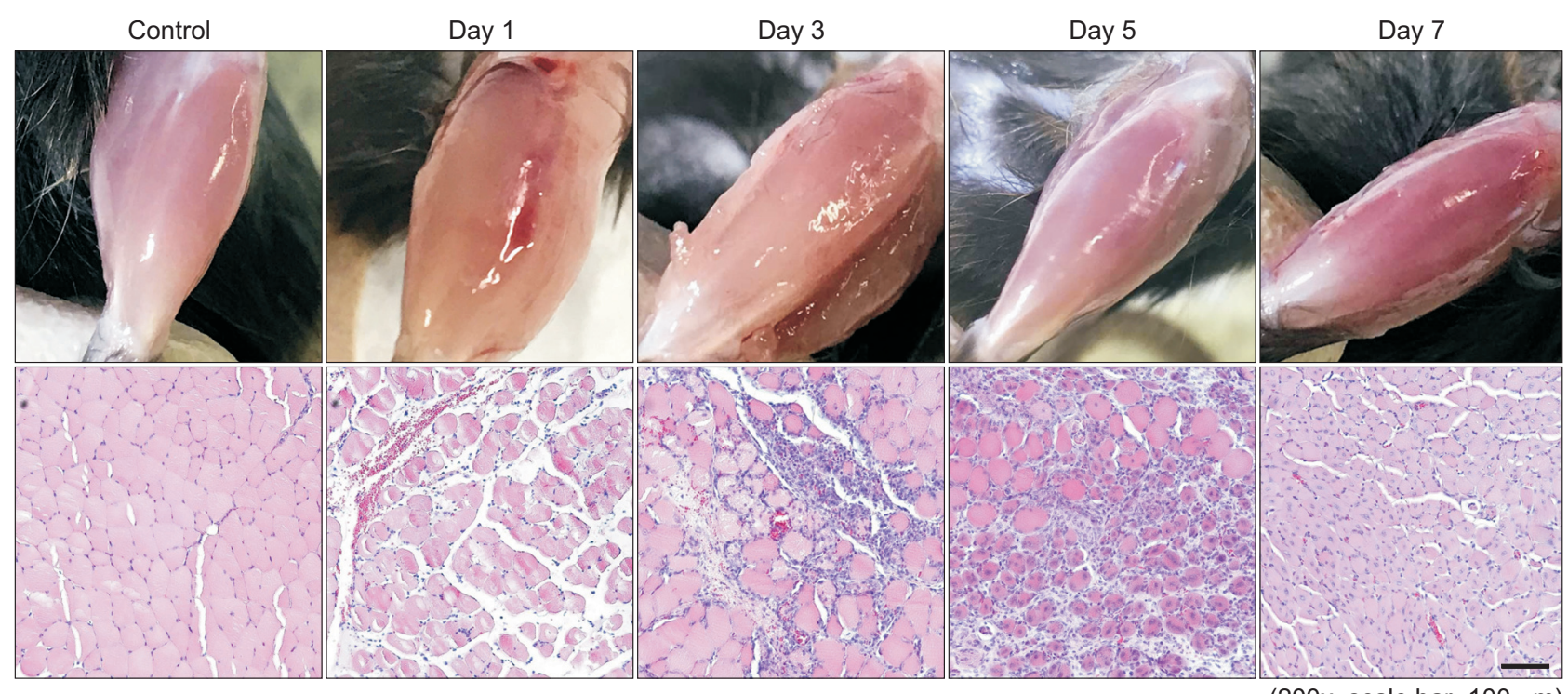

(200x, scale bar $=100 \mu \mathrm{m})$

Fig. 2. Representative gross morphology and hematoxylin and eosin-stained sections of tibialis anterior muscle at days $1,3,5$, and 7 after barium chloride $\left(\mathrm{BaCl}_{2}\right)$ injection, with uninjured muscle (control).

Table 1. Expression of selected genes from RNA sequencing, comparing tibialis anterior muscles at 7 days after $\mathrm{BaCl}_{2}$ injection to uninjured tibialis anterior muscles ( $\mathrm{n}=4$ for each group)

\begin{tabular}{|c|c|c|c|c|}
\hline Gene ID & Gene name & $\log _{2} \mathrm{FC}$ & $\log _{2} \mathrm{FC} \mathrm{SE}$ & $\mathrm{p}$-value \\
\hline \multicolumn{5}{|c|}{ Markers of proliferating satellite cells } \\
\hline ENSMUSG00000028736 & Pax7 & 1.91 & 0.28 & $2.41 \times 10^{-10}$ \\
\hline ENSMUSG00000000435 & Myf5 & 2.23 & 0.28 & $2.15 \times 10^{-13}$ \\
\hline ENSMUSG00000009471 & Myod1 & 0.42 & 0.24 & 0.163 \\
\hline ENSMUSG00000056493 & Foxk1 & -0.27 & 0.38 & 0.630 \\
\hline ENSMUSG00000026208 & Des & 0.34 & 0.28 & 0.374 \\
\hline \multicolumn{5}{|c|}{ Markers of differentiating satellite cells } \\
\hline ENSMUSG00000005320 & Fgfr4 & 2.14 & 0.31 & $1.64 \times 10^{-10}$ \\
\hline ENSMUSG00000020908 & Myh3 & 6.66 & 0.86 & $6.51 \times 10^{-13}$ \\
\hline ENSMUSG00000029467 & Atp2a2 & 1.70 & 0.48 & 0.002 \\
\hline ENSMUSG00000064179 & Tnnt1 & 2.27 & 0.52 & $9.93 \times 10^{-5}$ \\
\hline ENSMUSG00000048583 & $\operatorname{Ig} f 2$ & 5.94 & 0.30 & $1.09 \times 10^{-84}$ \\
\hline ENSMUSG00000027107 & Chrna1 & 2.36 & 0.25 & $4.55 \times 10^{-19}$ \\
\hline ENSMUSG00000028364 & Tnc & 4.61 & 0.61 & $2.40 \times 10^{-12}$ \\
\hline ENSMUSG00000026459 & Myog & 4.26 & 0.35 & $6.48 \times 10^{-32}$ \\
\hline
\end{tabular}

FC, fold change; SE, standard error. 


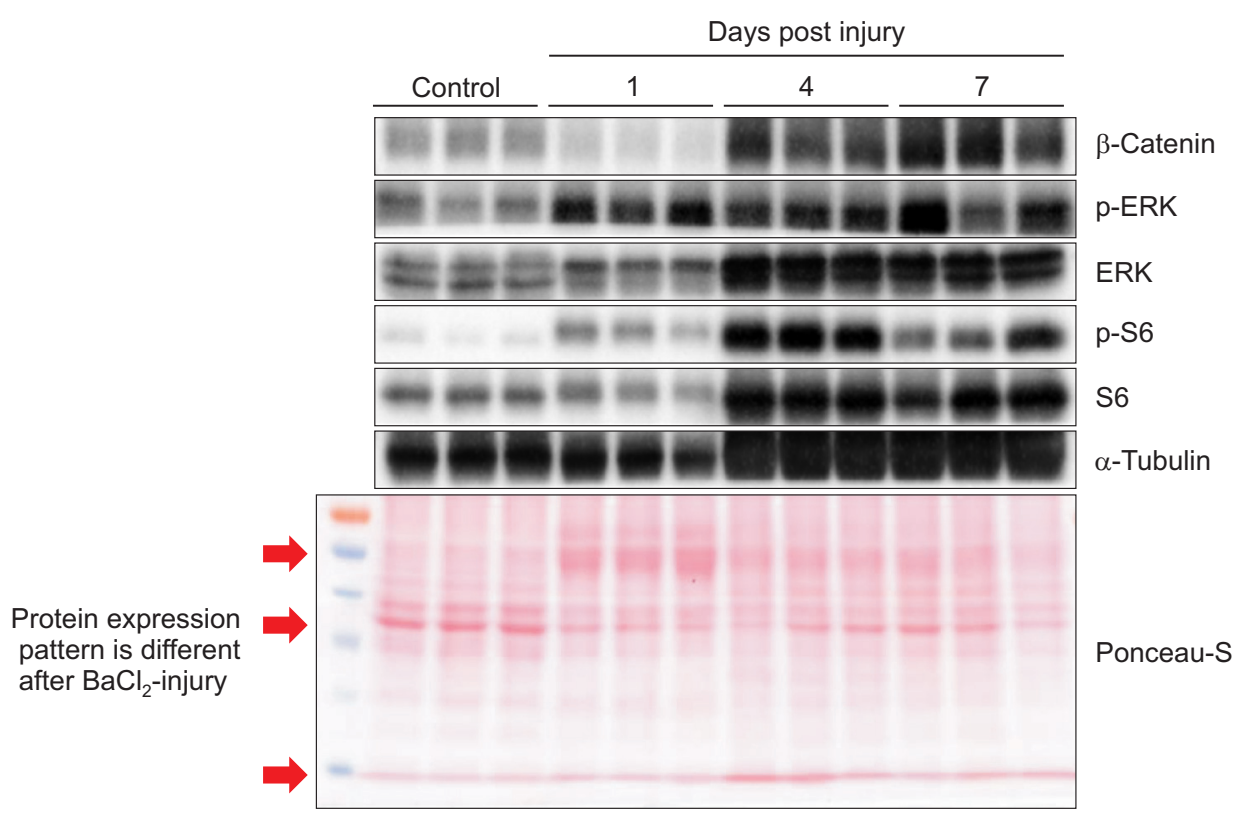

Fig. 3. Protein expression in tibialis anterior muscle at days 1,4 , and 7 after barium chloride $\left(\mathrm{BaCl}_{2}\right)$ injection compared to uninjured muscle (control), examined by western blotting. $\alpha-\mathrm{Tu}-$ bulin was used as the loading control ( $n=3$ for each group).
Igf2, Chrna1, Tnc, and Myog were consistently activated at day 7.

To assess cellular proliferation and protein synthesis activity at days 1, 4, and 7 after $\mathrm{BaCl}_{2}$ injection, western blot analysis was performed against proteins known to be activated during muscle regeneration. The levels of $\beta$-catenin, phospho- $\beta$-catenin, phospho-S6, and phospho-ERK were increased in the $\mathrm{BaCl}_{2}$-injected muscle at days 4 and 7 compared to those in control muscle (Fig. 3).

\section{DISCUSSION}

This study assessed the systemic and local phenotypes of the $\mathrm{BaCl}_{2}$-induced muscle injury model. Using varying $\mathrm{BaCl}_{2}$ doses to induce muscle injury, we found that the experimental window in terms of injection dosage was limited as acute mortality in 50\% of mice was observed when $100 \mu \mathrm{L}$ of a $1.2 \% \mathrm{BaCl}_{2}$ solution was injected into TA muscles. Also, systemic rhabdomyolysis was observed based on serum color and blood chemistry after $\mathrm{BaCl}_{2}-$ induced TA injury. Locally, the injury and regeneration processes of $\mathrm{BaCl}_{2}$ were largely similar to the previously reported phenotypes produced by injecting snake venoms.

In most biological studies, muscle injury and regeneration models have been proposed as a strictly tissuespecific, local method to study regenerating mechanisms focusing on muscle stem cells and their local environments. ${ }^{2,5)}$ Although many studies have analyzed the effect of nontissue autonomous factors such as aging, ${ }^{20)}$ endocrine alterations, ${ }^{21)}$ and systemic inflammation ${ }^{22)}$ on muscle regeneration models of laboratory animals, studies on the systemic effects of muscle injury have been mostly confined to human clinical studies. ${ }^{23)}$ As a result, the possible systemic effects of various muscle injury models in mice have been not been carefully assessed. In the present study, we observed two distinct systemic effects of $\mathrm{BaCl}_{2}$ injection model.

First, high-dose $\mathrm{BaCl}_{2}$ injection, with a two-fold increase in dosage, resulted in 50\% of the mice dying within 1 day after injection. Since $\mathrm{BaCl}_{2}$ is a known arrhythmogenic agent acting as potassium channel blocker with an LD50 of $19.2 \mathrm{mg} \mathrm{Ba}^{2+} / \mathrm{kg}$ in ICR mice, ${ }^{18,24)} 100 \mu \mathrm{L}$ of $1.2 \%$ $\mathrm{BaCl}_{2}$ corresponds to $31.8 \mathrm{mg} \mathrm{Ba} / \mathrm{kg}$, which might have been enough to cause fatal arrhythmia. Moreover, in standard experiments of $\mathrm{BaCl}_{2}$-induced muscle injury using 50 $\mu \mathrm{L}$ of a $1.2 \% \mathrm{BaCl}_{2}$ solution (15.9 $\mathrm{mg} \mathrm{Ba}^{2+} / \mathrm{kg}$ ), mice may suffer cardiac arrhythmias which may cause alterations in systemic physiology compared to that in uninjected control mice, although these arrhythmias may not lead to mortality. This effect on survival is likely specific to $\mathrm{BaCl}_{2}$ as other muscle injury methods do not report such systemic effects and the mechanisms of muscle injury are different.

Second, rhabdomyolysis-like features were observed in the analysis of serum at days 1 and 7 after $\mathrm{BaCl}_{2}$ injection. After centrifugation, the serum supernatants presented with a bright red color at days 1 and 7, with elevated serum CK and LDH levels at days 1 and 7, suggesting myoglobulinemia with myonecrosis similar to what is observed in clinical snake venom poisoning. ${ }^{25)}$ Although the possibility of acute kidney injury was low, with serum creatinine levels not elevated significantly by $\mathrm{BaCl}_{2}$ injection, myoglobinemia may cause systemic responses that may eventually affect the muscle regeneration process, which requires the precise orchestration of inflammatory cells, local paracrine environment, and muscle stem cells. ${ }^{26,27)}$ These systemic effects are unavoidable in in vivo muscle injury models since all muscle injury and regeneration models accompany acute, severe myonecrosis in the mouse hindlimb.

Local phenotypic characterization of injury and regen- 
eration after $\mathrm{BaCl}_{2}$ injection revealed temporal changes in histological features similar to those in previous models of muscle regeneration, with suppurative inflammation at day 1 , cellular proliferation at day 3, myotube formation at day 5, and myotube maturation at day 7 and beyond. ${ }^{5,15,28)}$ Assessment of protein and RNA expressions at day 7 showed upregulation of differentiation and maturation markers in accordance with previous reports. ${ }^{5,15)}$ Based on these findings, $\mathrm{BaCl}_{2}$-induced muscle injury models are largely similar to other injury models for the study of muscle regeneration.

Although we identified the systemic effects of the Ba$\mathrm{Cl}_{2}$-induced muscle injury model that were less considered in previous studies, this study has several limitations. First, we were not able to directly compare $\mathrm{BaCl}_{2}$ to cardiotoxin, the most popular venom used in muscle injury experiments, due to logistic issues with local regulations. Also, we did not confirm that the acute mortality after highdose $\mathrm{BaCl}_{2}$ injection was actually due to fatal arrhythmia. This limiting aspect may be resolved by performing electrocardiogram studies during the acute observation periods after $\mathrm{BaCl}_{2}$ injection.

However, with clinical and systemic perspectives on muscle regeneration, we observed that muscle injury and regeneration following $\mathrm{BaCl}_{2}$ injection occurred with possible efferent signals that may influence other organs, which may, in turn, cause indirect effects on muscle regeneration phenotypes. In particular, the effects of crosstalk between injured/repairing muscle and other organ systems should be considered when studying alterations of muscle regeneration within the context of aging.

\section{CONFLICTS OF INTEREST DISCLOSURES}

The researchers claim no conflicts of interest.

\section{ACKNOWLEDGMENTS}

This study was supported by a Korean Geriatrics Society Young Researcher Grant (2016) and National Research Foundation of Korea grants NRF-2018R1A2A3075389 and NRF-2016R1A2B1011083.

\section{REFERENCES}

1. Krafts KP. Tissue repair: the hidden drama. Organogenesis 2010;6:225-33.

2. Blau HM, Cosgrove BD, Ho AT. The central role of muscle stem cells in regenerative failure with aging. Nat Med 2015;21:854-62.

3. Kim BH, Jung HW, Seo SH, Shin H, Kwon J, Suh JM. Synergistic actions of FGF2 and bone marrow transplantation mitigate radiation-induced intestinal injury. Cell Death Dis 2018;9:383.

4. Gayraud-Morel B, Chrétien F, Tajbakhsh S. Skeletal muscle as a paradigm for regenerative biology and medicine. Regen Med 2009;4:293-319.

5. Chargé SB, Rudnicki MA. Cellular and molecular regulation of muscle regeneration. Physiol Rev 2004;84:209-38.

6. Sousa-Victor P, Gutarra S, García-Prat L, Rodriguez-Ubreva J, Ortet L, Ruiz-Bonilla V, et al. Geriatric muscle stem cells switch reversible quiescence into senescence. Nature 2014;506:316-21.

7. Cesari M. Physical frailty and sarcopenia: development of a framework for supporting interventions against incident mobility disability. Ann Geriatr Med Res 2017;21:42-8

8. Jang HC. How to diagnose sarcopenia in Korean older adults? Ann Geriatr Med Res 2018;22:73-9.

9. Park HM. Current status of sarcopenia in Korea: a focus on Korean geripausal women. Ann Geriatr Med Res 2018;22:52-61.

10. Cruz-Jentoft AJ, Bahat G, Bauer J, Boirie Y, Bruyère 0 , Cederholm T, et al. Sarcopenia: revised European consensus on definition and diagnosis. Age Ageing 2019;48:16-31.

11. Conboy IM, Conboy MJ, Smythe GM, Rando TA. Notch-mediated restoration of regenerative potential to aged muscle. Science 2003;302:1575-7.

12. Carlson ME, Suetta C, Conboy MJ, Aagaard P, Mackey A, Kjaer $\mathrm{M}$, et al. Molecular aging and rejuvenation of human muscle stem cells. EMBO Mol Med 2009;1:381-91.

13. Brack AS, Conboy MJ, Roy S, Lee M, Kuo CJ, Keller C, et al. Increased Wnt signaling during aging alters muscle stem cell fate and increases fibrosis. Science 2007;317:807-10.

14. Brack AS, Conboy IM, Conboy MJ, Shen J, Rando TA. A temporal switch from notch to Wnt signaling in muscle stem cells is necessary for normal adult myogenesis. Cell Stem Cell 2008;2:50-9.

15. Carosio S, Berardinelli MG, Aucello M, Musarò A. Impact of ageing on muscle cell regeneration. Ageing Res Rev 2011;10:35-42.

16. Kim JH, Han GC, Seo JY, Park I, Park W, Jeong HW, et al. Sex hormones establish a reserve pool of adult muscle stem cells. Nat Cell Biol 2016;18:930-40.

17. Yanagisawa T, Hashimoto $H$, Taira N. Interaction of potassium channel openers and blockers in canine atrial muscle. Br J Pharmacol 1989;97:753-62.

18. Syed IB, Hosain F. Determination of LD50 of barium chloride and allied agents. Toxicol Appl Pharmacol 1972;22:150-2.

19. Fossa AA, Carlson GP. Antiarrhythmic effect of disulfiram in various cardiotoxic models. Pharmacology 1983;26:164-71.

20. Conboy IM, Conboy MJ, Wagers AJ, Girma ER, Weissman IL, Rando TA. Rejuvenation of aged progenitor cells by exposure to a young systemic environment. Nature 2005;433:760-4.

21. Akhmedov D, Berdeaux R. The effects of obesity on skeletal muscle regeneration. Front Physiol 2013;4:371.

22. Langen RC, Schols AM, Kelders MC, van der Velden JL, Wouters EF, Janssen-Heininger YM. Muscle wasting and impaired muscle regeneration in a murine model of chronic pulmonary inflammation. Am J Respir Cell Mol Biol 2006;35:689-96.

23. Better OS, Rubinstein I, Reis DN. Muscle crush compartment syndrome: fulminant local edema with threatening systemic effects. Kidney Int 2003;63:1155-7.

24. Mattila MJ, Anyos K, Puisto EL. Cardiotoxic actions of doxepin and barium chloride in conscious rabbits. Arch Toxicol Suppl 1986;9:205-8.

25. Azevedo-Marques MM, Cupo P, Coimbra TM, Hering SE, Rossi MA, Laure CJ. Myonecrosis, myoglobinuria and acute renal failure induced by South American rattlesnake (Crotalus durissus terrifi- 
cus) envenomation in Brazil. Toxicon 1985;23:631-6.

26. Brack AS, Rando TA. Tissue-specific stem cells: lessons from the skeletal muscle satellite cell. Cell Stem Cell 2012;10:504-14.

27. Shea KL, Xiang W, LaPorta VS, Licht JD, Keller C, Basson MA, et al. Sprouty1 regulates reversible quiescence of a self-renewing adult muscle stem cell pool during regeneration. Cell Stem Cell 2010;6:117-29.

28. Hardy D, Besnard A, Latil M, Jouvion G, Briand D, Thépenier C, et al. Comparative study of injury models for studying muscle regeneration in mice. PLoS One 2016;11:e0147198. 